\title{
Improved Detection Limits for Electrospray Ionization on a Magnetic Sector Mass Spectrometer By Using an Array Detector
}

\author{
Robert B. Cody, Jun Tamura, Jeffrey W. Finch, and Brian D. Musselman \\ JFOI. UISA, Inc., Peabody, Massachusetts, USA
}

\begin{abstract}
Array detection was compared with point detection for solutions of hen egg-white lysozyme, equine myoglobin, and ubiquitin analyzed by electrospray ionization with a magnetic sector mass spectrometer. The detection limits for samples analyzed by using the array detector system were at least 10 times lower than could be achieved by using a point detector on the same mass spectrometer. The minimum detectable quantity of protein corresponded to a signal-to-background ratio of approximately 2:1 for a $500 \mathrm{amol} / \mu \mathrm{L}$ solution of hen egg-white lysozyme. However, the ultimate practical sample concentrations appeared to be in the $10-100 \mathrm{fmol} / \mu \mathrm{L}$ range for the analysis of dilute solutions of relatively pure proteins or simple mixtures. (J Am Soc Mass Spectrom 1994, 5, 194-200)
\end{abstract}

$\mathrm{E}$ lectrospray ionization (ESI) [1-7] sources have been successfully interfaced to all common types Jof mass analyzers including quadrupole [3-8], time-of-flight $[9,10]$, ion trap [11], Fourier transform [12-16], and magnetic sector mass spectrometers [17-25]. The low detection limits often reported for analyses with ESI may result from the relatively low chemical background and from an enhanced detector response to multiply charged ions that have been accelerated to relatively high kinetic energies.

The use of the ESI method for detection of small quantities of analyle is described in the recent report by Wahl et al. [26] of the detection of amole-level quantities of a series of intermediate molecular weight proteins such as cytochrome $c$, myoglobin, and carbonic anhydrase. These experiments were performed by using a scanning quadrupole mass spectrometer equipped with an ESI source and a chemically modified capillary electrophoresis (CE) column designed to permit sampling of small volumes of the analyte solution. The experimental protocol called for the use of a $30-\mu \mathrm{M}$ solution of the proteins from which a small volume of sample was transferred onto a CE column. An estimated 600 amol quantity of each component of the mix was analyzed after separation by CE. It is difficult to define a true practical detection limit for the CE work because the experimental procedure requires that the sample be introduced onto the $\mathrm{CE}$ column from a highly concentrated solution. Although it is not yet feasible to handle total quantities of proteins in the amole range, this work demonstrates that very small quantities of proteins can be ionized and detected on a

Address reprint requests to Robert B. Cody, JEOL USA, Inc., 11 Dearborn Road, Peabody, MA 01960. routine basis by using $\mathrm{CE} / \mathrm{ESI}$ mass spectrometry techniques.

Other approaches are being investigated to reduce the detection limits for analyses with ESI coupled with various types of mass spectrometers. For example, Mordehai and Henion [27] have reported the coupling of CE with ion spray (pneumatically assisted electrospray) and an ion-trap mass spectrometer for the detection of small molecules. A signal-to-noise ratio of 15 was reported for an injection of $\mathbf{1 5 0} \mathrm{pg}$ (approximately $670 \mathrm{fmol}$ ) of neostigmine. Verentchikov et al. [10] have reported detection limits in the low femtomole range for proteins analyzed by ESI coupled with a time-offlight mass spectrometer. Fourier transform mass spectrometry (FTMS) has demonstrated excellent resolution for ESI [12-14], and it has a potential for the detection of small numbers of multiply charged ions [15]. Some promising initial reports have appeared that suggest relatively low detection limits for FTMS. A recent example is the report by Guan et al. [16] showing the detection of femtomole-level quantities of proteins. However, these figures appear to reflect an estimate based upon duty cycle of the number of ions injected into the mass spectrometer from a 3-10 $\mathrm{pmol} / \mu \mathrm{L}$ solution. The limiting detection limits for ESI coupled with FTMS may ultimately be determined by improvements in sample handling and duty cycle.

Efforts to use ESI sources for magnetic sector mass spectrometers have emphasized the use of these instruments for high-resolution and accurate mass measurements [17-23]. For example, we have recently reported the use of a magnetic sector mass spectrometer to provide high-resolution and accurate mass measurements for a variety of compounds analyzed by ESI [22]. The present work attempts to examine the sensi- 
tivity and detection limits for ESI coupled with magnetic sector mass spectrometry.

Generally, the detection limits obtained with magnetic sector mass spectrometers have not exceeded the limits observed with quadrupole mass spectrometers when both instruments are operated in the scanning mode with point detectors. The difference in sensitivity is negligible for molecules with molecular weights of less than 25,000. Electrospray (and ion spray) on both magnetic sector and quadrupole instruments can often be used to detect analytes in the $100 \mathrm{pmol} / \mu \mathrm{L}$ to $10 \mathrm{fmol} / \mu \mathrm{L}$ concentration range. However, a decrease in resolution has been observed for large $(>30,000 \mathrm{Da})$ proteins analyzed with the magnetic sector mass spectrometer [28]. This was attributed to a difference in collision cross sections for the larger molecules, and this effect may hinder the detection of trace quantities of large proteins with a magnetic sector mass spectrometer.

A few preliminary reports have appeared on the use of array detection [29-33] to improve the detection limits for magnetic sector mass spectrometers [23-25]. We have reported preliminary results for the ESI analysis of several proteins by using a magnetic sector mass spectrometer equipped with an array detector. Our preliminary investigations indicated that it is possible to detect positive multiply charged ions from subfemtomole quantities of proteins by using array detection [23]. The minimum detectable quantity corresponded to a signal-to-background ratio of 2:1 for 1 fmol consumed from a $500 \mathrm{amol} / \mu \mathrm{L}$ solution of hen egg-white lysozyme. Dobberstein et al. [24] have reported detection limits for negative ions from several small molecules analyzed by using a magnetic sector mass spectrometer equipped with a position and time-resolved ion counting detector. In this work, a minimum sample consumption of $1 \mathrm{fmol}$ was reported for a singly charged species, and a minimum sample consumption of $250 \mathrm{fmol}$ was reported for a multiply charged species. Bateman et al. [25] have also reported product-ion mass spectra obtained by using a tandem mass spectrometer equipped with an array detector. However, no detection limits were reported for that work.

As is evident from the efforts cited above, there is wide disparity in the reported sensitivity for ESI which can often be traced to the different sample introduction methods, mass analysis conditions, and other variations in experimental protocol. The detection limits for ESI are often reported in terms of the amount of analyte consumed during the measurement period. That is, a known analyte concentration is continuously introduced, ionized, mass analyzed, and detected over a specific time period. The product of analyte concentration, flow rate, and analysis time is used to calculate the sample consumption. We have adopted this convention for the detection limits reported in this article. However, the analyte solutions were introduced by using a flow injector to restrict the sample consumption to the time period required for mass analysis.
The following report offers a closer examination of the detection limits for ESI with a magnetic sector mass spectrometer equipped with an array detector. Proteins are used as model compounds to study the detection limits for multiply charged compounds. A direct comparison is made between the point detector and the array detector for ESI protein analyses, and practical limits for handling dilute solutions of analyte are considered.

\section{Experimental}

\section{Mass Spectrometer}

Mass spectra were acquired with a JMS-HX110A (JEOL Ltd., Akishima, Japan) forward-geometry doublefocusing mass spectrometer equipped with an ESI source (Analytica of Branford, Branford, CT). The electrospray ion source is identical to the source described in a previous report from our laboratory [22]. The mass spectrometer has a maximum acceleration potential of $7 \mathrm{kV}$ when operated in the ESI mode.

The mass spectrometer was operated at an acceleration voltage of $5 \mathrm{kV}$. The mass spectrometer main slit width was adjusted to the theoretical value for a resolving power of 1700 (full width at half maximum definition), and the intermediate slits were fully opened. In our experience, the actual measured resolution values for a given slit setting differ from the theoretical values by only a few percent. All mass spectra were acquired by using a single analysis, without averaging of multiple spectra. Prior to installing the electrospray ion source, the mass spectrometer was calibrated with a (perfluoroalkyl) phosphazine mixture at a $5-\mathrm{kV}$ acceleration potential in positive-ion and negative-ion fast-atom bombardment modes. These static calibrations (acquired at a scan rate of $90 \mathrm{~s} / \mathrm{scan}$ ) were used to step the magnet to the center mass values required for proper mass assignment with the array detector system.

\section{Array Detector}

The mass spectrometer ion optics have been modified by the addition of a quadrupole/octapole/quadrupole lens assembly (referred to as a quadrupole doublet or " $\mathrm{QPD}$ ) which permits the manipulation of the double-focusing objective of the spectrometer. The commercial implementation of the array detector system is similar to that described by Hill et al. [29], and the reader is referred to that work for a detailed description of the lens system.

The array detector dispersion is adjustable to provide simultaneous detection over a mass range of approximately $5 \%$ to $25 \%$ of the center mass. For example, at a $10 \%$ dispersion and a center mass of 400 $\mathrm{Da}$, the mass range that can be simultaneously detected is $40 \mathrm{Da}$. Increasing the dispersion to $20 \%$ per- 
mits the simultaneous detection of an $80 \mathrm{Da}$ mass range at the same center mass setting. The dispersion is selected by adjusting the array detector angle and the potentials on the quadrupole doublet ion optic elements. The array angle and potentials of the ion optic elements are under computer control. The mass dispersion at a particular angle is determined experimentally by using a reference standard compound such as CsI or perfluoroalkylphosphazine, and this dispersion measurement is used together with the magnet stepping center mass tables to calibrate the mass range.

For the experiments in this report, the variable dispersion array detector was set to a fixed $10 \%$ dispersion. Mass spectra were acquired by setting the mass spectrometer magnet field to jump from successive center mass values which differ in mass by $10 \%$ with a small overlap (typically 1\%) to ensure continuity between segments. The last segment is a dark-current segment acquired with no ion current hitting the detector. The magnet stepping is controlled by the computer based upon a previously acquired slow-scan ("static") calibration. Typical spectrum acquisition involves the collection of a sequence of $0.5 \mathrm{~s}$ exposures of the photodiode array collected at each mass interval. Following acquisition of each of the sequential segments of the mass spectra, the segments are automatically gain-corrected, dark-current subtracted, and concatenated to create a single continuous mass spectrum.

\section{Electrospray Ionization Conditions}

The analyte was dissolved in a 1:1 water/methanol solution containing glacial acetic acid $(2 \%)$. The solvents used for the dilutions were stored in Teflon-lined vials. Serial tenfold dilutions were made from $100 \mu \mathrm{M}$ stock solutions to provide solutions with the proper analyte concentration, and the most dilute samples were analyzed immediately after the dilution was prepared. The samples were diluted into polycarbonate tubes which had been previously washed with the electrospray solvent. The sample was introduced into the ESI source either by infusion or by flow injections with a $20 \mu \mathrm{L}$ loop irjector. A syringe pump (Model 210, Harvard Apparatus, South Natick, MA) provided a constant $2 \mu \mathrm{L} / \mathrm{min}$ flow rate for infusion or loop injections. The nitrogen drying gas in the ESI source was maintained at $145^{\circ} \mathrm{C}$ at a flow rate of approximately $5 \mathrm{~L} / \mathrm{min}$ for all ESI measurements.

Contaminants and interferences can cause problems for the detection of very small quantities of analyte, as evident from the relatively large interference peaks in the low mass region of many of the ESI/array detector mass spectra. The sample containers must be carefully washed with the electrospray solvent solution, and the most dilute analyte solutions should be analyzed immediately after preparation. Otherwise, sample losses due to adsorption of the analyte to the container walls can result in poor detection limits, and contaminants can obscure the detection of an unknown analyte.

\section{Scan Conditions and Acquisition Times}

The magnet was scanned for the point detector experiments at a scan speed of 37 seconds to cover the 500-3000 Da mass range, with a 3 second dead time between scans. Two scans were added to provide a mass spectrum that was equivalent to an 80 -second total analysis time. The magnet stepping conditions for the array detector experiment were set to cover the 500-3000 Da mass range in 24 segments at a fixed $10 \%$ dispersion. A 1-second settling time allowed the magnet to completely stabilize between magnet steps. The array detector integration time was adjusted to 2.2 seconds for a total analysis time of 80 seconds. No significant information was observed in the mass range above $2000 \mathrm{Da}$, and therefore that section of the mass spectrum is not presented.

\section{Results and Discussion}

\section{Detection Limits for Infusions of Protein Solutions}

The detection limits were determined for the point detector and the array detector for solutions containing equine myoglobin, which has an average molecular weight of $16951.5 \mathrm{Da}$ [34]. The ESI mass spectra obtained from the analysis of $3 \mathrm{pmol} / \mu \mathrm{L}(8 \mathrm{pmol}$ consumed during data acquisition) and $300 \mathrm{fmol} / \mu \mathrm{L} \mathrm{(800}$ fmol consumed during data acquisition) solutions of myoglobin with the point detector are shown in Figure $1 a$ and $b$, respectively. The major peaks in the spectrum correspond to the expected multiply charged ion species ranging from $[\mathrm{M}+13 \mathrm{H}]^{13+}$ to $[\mathrm{M}+21 \mathrm{H}]^{21+}$ for this protein. The ions near $m / z 616$ correspond to the singly charged, dissociated heme group. The charge state tends toward more highly charged species for the more dilute solutions; this is consistent with our experience for other proteins $[22,23]$. The signal-to-noise ratios for the mass spectra are approximately $25: 1$ and 13:1, respectively. These values are estimated by using the most prominent peak to define the signal intensity and twice the standard deviation of the background level over a 50 Da region near that peak as an estimate of the noise.

The ESI mass spectra obtained from the array detector analysis of the $300 \mathrm{fmol} / \mu \mathrm{L}$ and $30 \mathrm{fmol} / \mu \mathrm{L}$ solutions are shown in Figure $1 \mathrm{c}$ and $\mathrm{d}$. The amount of analyte consumed during acquisition was $800 \mathrm{fmol}$ and $80 \mathrm{fmol}$, respectively. The signal-to-noise ratios for these mass spectra are $31: 1$ and $13: 1$, respectively, estimated by using the same criteria applied to the point detector mass spectra. The more dilute solution shows the same trend toward increased charging that was described above for the point detector mass spectra. The ESI mass spectrum for the solvent blank (Figure 1e) shows that the large singly charged ion at $m / z$ 538 is a solvent-related contaminant. This solvent blank was obtained from the same solvent bottle that was used for the point detector measurements.

Note that $300 \mathrm{fmol} / \mu \mathrm{L}$ was the most dilute solution analyzed by using the point detector, while 300 

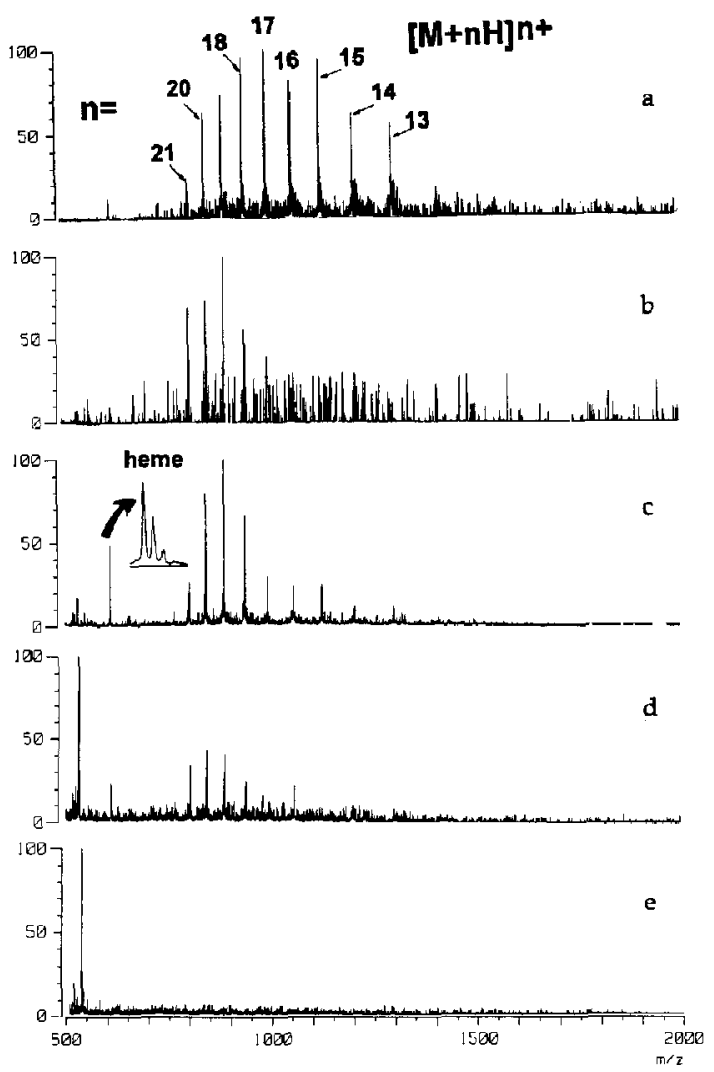

Figure 1. ESI mass spectra for infusions of myoglobin at solution concentrations of (a) $3 \mathrm{pmol} / \mu \mathrm{L}$ (point detector), (b) 300 $\mathrm{fmol} / \mu \mathrm{L}$ (point detector), (c) $300 \mathrm{fmol} / \mu \mathrm{L}$ (array detector), (d) $30 \mathrm{fmol} / \mu \mathrm{L}$ (array detector), and (e) solvent blank (array detector).

fmol $/ \mu \mathrm{L}$ was the most concentrated solution examined by using the array detector. A comparison of the 3 pmol $/ \mu \mathrm{L}$ point-detector mass spectrum (Figure 1a) with the $300 \mathrm{fmol} / \mu \mathrm{L}$ array-detector mass spectrum (Figure 1c) shows that the array detector is approximately 10 times more sensitive than the point detector for these experiments. This estimate is supported by a comparison of the signal-to-noise ratios for the 300 $\mathrm{fmol} / \mu \mathrm{L}$ point-delector mass spectrum and the 30 $\mathrm{fmol} / \mu \mathrm{L}$ array detector mass spectrum (Figure $\mathrm{lb}$ and d).

The low-mass peaks, including the background peaks and peaks for the heme group are very weak in the point-detector mass spectra, but they are quite prominent in the array detector mass spectra. This may be due to the use of postacceleration with the point detector, which reduces the natural sensitivity bias against heavy ions. The array detector does not make use of postacceleration, and therefore the array detector may exaggerate the response to low-mass ions: It is significant that the isotopic peaks are resolved for the heme species in the array-detector mass spectrum of the $30 \mathrm{fmol} / \mu \mathrm{L}$ solution. The measured resolution for the isotope peaks is 1600 (full width at half maximum definition), which is in reasonable agreement with the theoretical resolving power of 1700 for the slit settings and the $10 \%$ dispersion setting for the array detector. The resolution of individual isotopic peaks can be very helpful in distinguishing small singly or doubly charged species from the multiply charged species arising from larger molecules, and this information can greatly facilitate the interpretation of the ESI mass spectrum of an unknown at relatively low concentrations where interferences may be present.

The minimum detectable quantity of myoglobin was determined by analyzing a $3 \mathrm{fmol} / \mu \mathrm{L}$ solution and a total sample consumption of $8 \mathrm{fmol}$. The mass spectrum is shown in Figure 2. Three peaks can be clearly observed that correspond to the expected mass-tocharge ratios for the species having 19-21 charges. The presence of multiple charge states for proteins can often allow one to recognize these peaks in the presence of impurities that have only one charge state. It should be noted that an unrelated contaminant peak appearing at $m / z 977$ is nearly as abundant as the myoglobin peaks. This peak does not appear in the solvent blank, and its appearance in the myoglobin spectrum is attributed to trace contamination during the dilution of the analyte. The ESI mass spectrum was transformed by using the algorithm of Mann et al. [3] to produce the mass spectrum shown in the inset for Figure 2. The transformed mass spectrum combines the signal from each charge state into a single peak that corresponds to the mass of the uncharged molecular species. The transformation is especially helpful for interpreting ESI mass spectra near the detection limits because the transformation sums the intensities for the different charge states. This emphasizes the signal from several multiply charged species from the protein relative to singly charged interference ions such as the $m / z 538$ and 977 peaks, although it should be recog-

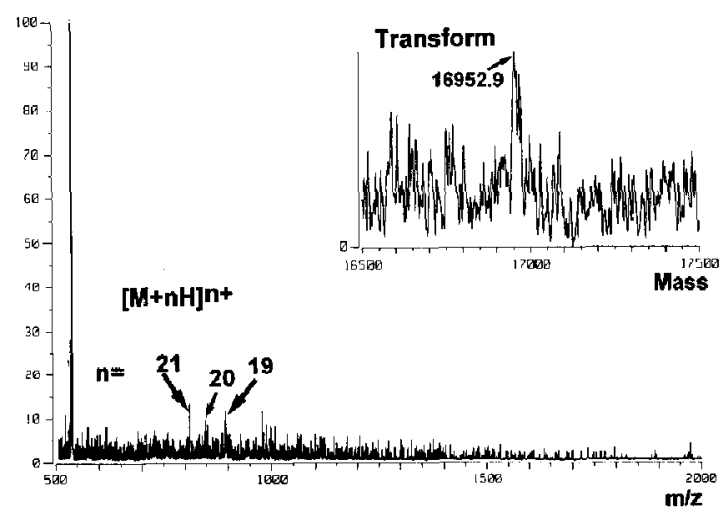

Figure 2. ESI mass spectrum acquired with the array detector for infusion of a $3 \mathrm{fmol} / \mu \mathrm{L}$ solution of equine myoglobin. Inset: Transformation of the mass spectrum by the "deconvolution" algorithm of Marn et al. [3]. 
nized that this emphasis can be lost for very complex mixtures [35]. The molecular weight was determined from the transformed mass spectrum to be $16952.9 \mathrm{Da}$, which differs by only 1.4 Da from the theoretical molecular weight. The peak appears to contain two other components. If these are real species, then the masses are approximately $16 \mathrm{Da}$ and $22 \mathrm{Da}$ higher than the main component suggesting a species with an oxidized methionine and a sodium adduct. However, it is equally likely that these are artifacts due to the low signal-to-noise ratio.

Similar detection limits were observed for other protein solutions. Serial tenfold dilutions were made for hen egg-white lysozyme (average molecular weight $14,305.16 \mathrm{Da}$ [22]) starting with a $10 \mathrm{pmol} / \mu \mathrm{L}$ lysozyme stock solution. Mass spectra were acquired starting with the most dilute solution and ending with the most concentrated solution. The ESI mass spectra obtained by using the array detector are shown in Figure $3 a-d$, and the mass spectrum of the solvent blank is shown in Figure 3e. Note that the $m / z 538$ contaminant present in the solvent blank for the myoglobin dilutions was absent from the solvent blank for the lysozyme dilutions. This is attributed to the fact that the myoglobin and lysozyme measurements were taken several months apart and different bottles of solvent were used in preparing the solutions. The signal-to-background ratio obtained from the analysis of the $100 \mathrm{fmol} / \mu \mathrm{L}$ solution by using the point detector (not shown) was approximately 5:1 while the 10 $\mathrm{fmol} / \mu \mathrm{L}$ solution analyzed by using the array detector (Figure 3c) had a signal-to-background ratio in excess of 10:1. These measurements were repeated three times over the course of one year by using different array detector assemblies and electrospray ion sources, and the same detection limits were observed each time.

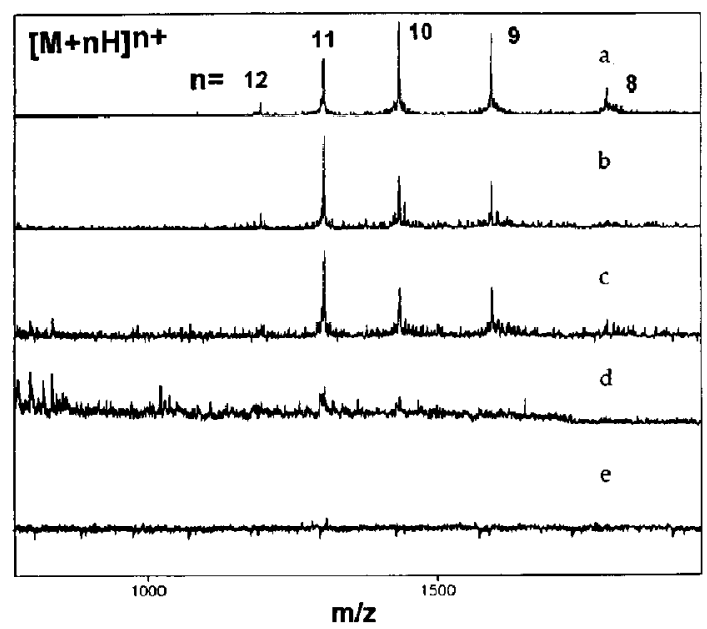

Figure 3. ESI mass spectra acquired with the array defector for infusions of hen egg-white lysozyme at solution concentrations of

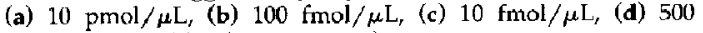
amol $/ \mu \mathrm{L}$. and (e) (a) (eolvent blank).
It should be noted that a minimum of two charge states must be observed to determine the molecular weight from multiply charged species in the mass spectrum of an unknown. The minimum detectable quantity of lysozyme was determined for array detection of a $500 \mathrm{amol} / \mu \mathrm{L}$ solution and a total sample consumption of approximately $1 \mathrm{fmol}$. Although the peaks are near the noise level and have poor peak shapes, the $[\mathrm{M}+10 \mathrm{H}]^{101}$ and $[\mathrm{M}+11 \mathrm{H}]^{11+}$ species are clearly discernible (Figure $3 \mathrm{~d}$ ). At this level, several contaminant peaks appear that are as abundant as the lysozyme peaks. However, the unresolved isotope distributions result in wider peaks for the multiply charged lysozyme species than are observed for singly charged low-mass interferences. Although this mass spectrum illustrates the minimum detectable quantity of lysozyme and it clearly indicates the presence of the known protein, it is doubtful that a mass spectrum with this signal-to-noise level would be useful in establishing the identity of an unknown. Note that the slightly better detection limits for lysozyme compared to myoglobin may result from the narrower charge distribution for lysozyme. We normally observe three to five prominent charge states for ESI of hen egg-white lysozyme, and we normally observe four to sixteen charge states for equine myoglobin [22]. The signal-tonoise ratio for the peaks for individual charge states will be reduced if the total ion current from the analyte is distributed over a large number of charge states.

\section{Sample Consumption and the Handling of Small Sample Quantities}

The detection limits reported in the preceding discussion were obtained under optimal conditions. That is, the analyte solution was introduced by infusion and the mass spectrometer and ion source were tuned to provide the maximum ion signal just prior to the acquisition of the mass spectra. This procedure may not be possible for the analysis of limited quantities of an unknown. The normal procedure is to optimize the mass spectrometer performance for a reference standard compound and to use these conditions for the analysis of the unknown component. This approach was tested by tuning the mass spectrometer and the electrospray ion source to maximize the signal for a $100 \mathrm{fmol} / \mu \mathrm{L}$ solution of hen egg-white lysozyme. These conditions were then used without any further tuning or readjustment to analyze a simulated "unknown" solution containing $20 \mathrm{fmol} / \mu \mathrm{L}$ of ubiquitin (average molecular weight $8564.9 \mathrm{Da}$ ).

The resulting mass spectrum shown in Figure 4 contains several peaks that can be readily assigned to the multiply charged species for ubiquitin. However, the low-mass region also contains very large singly charged peaks resulting from the chemical background. The charge state of these peaks can be easily determined by examining the separation between adjacent isotopic peaks (to be discussed below). Contami- 
nant peaks at higher mass-to-charge ratios values, such as the peak near $m / z 1500$, do not show multiple charge states and can be recognized as distinct species from the multiply charged proteins. These interference peaks are believed to originate from contaminants remaining in the plastic vials used to prepare the sample dilutions. The observation of these peaks emphasizes the need for contamination-free containers and solvents when the detection of trace-level amounts of analyte is desired. This concern has led to the development in our laboratory of a stricter sample handling protocol for trace analyses by ESI and array detection. In general, the multiply charged species produced by ESI of the protein solutions tend to have mass-to-charge ratios that are higher than those of the major contaminant ions. Nevertheless, the contribution of chemical interferences to the background "noise" level cannot be dismissed.

The definition of detection limits in terms of the "amount consumed" may be misleading in describing the minimum sample quantity that is required to obtain a useful molecular weight. A relatively large amount of sample may be introduced during sample introduction by infusion. The definition in terms of "amount consumed" also ignores sample losses that can occur in the dead volume of the plumbing connections to the electrospray interface. To test the utility of the array detector for the analysis of trace levels of proteins under conditions that simulate the high sample volumes of a routine service laboratory, we measured equine myoglobin without any optimization or retuning after the mass spectrometer had been run in electrospray mode for 8 hours. A loop injection of 10 $\mu \mathrm{L}$ of a $44 \mathrm{fmol} / \mu \mathrm{L}$ solution of equine myoglobin gave the mass spectrum shown in Figure 5. The time

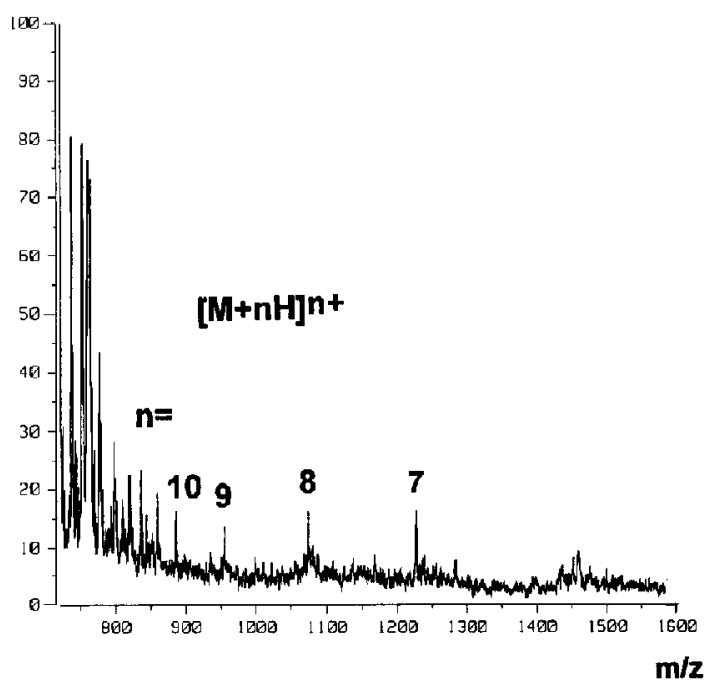

Figure 4. ESI mass spectra acquired with the array detector for infusion of a $20 \mathrm{fmol} / \mu \mathrm{L}$ solution of ubiquitin. Sample consumption was $20 \mathrm{fmol}$. required for the analyte to reach the mass spectrometer was determined for a reference compound, and the mass spectrum was acquired with the array detector by integrating over the entire elution time ( 5 minutes) for the analyte. The total amount of sample injected was $\mathbf{4 4 0} \mathrm{fmol}$. Seven peaks that correspond to multiply charged species from myoglobin can be detected above the noise level. The presence of seven consecutive charge states for myoglobin makes it possible to identify the multiply charged protein in spite of the presence of interference peaks in this mass range that do not exhibit multiple consecutive charge states. Transformation of the mass spectrum by the algorithm of Fenn and co-workers [3] gave the mass spectrum shown in the inset in Figure 5. The $\mathbf{4 4 0} \mathrm{fmol}$ total sample quantity probably provides a more realistic estimate than the estimate reported for the example in Figure 2 of $8 \mathrm{fmol}$ "amount consumed" as the minimum total amount of sample that would be required for an analysis.

It is our opinion that, in addition to the type of detector used, the major factors that determine the detection limits for these experiments are the chemical background, the ionization efficiency of the electrospray source, and the transmission of the ions from the ESI source into the mass spectrometer. Some reduction in the detection limits may be possible by increasing the array detector dispersion to permit integration over a wider mass range at the expense of resolution. This will permit faster spectrum acquisition and/or longer integration times. Reducing the number of segments required to cover the desired mass range can significantly reduce the time requirements and sample consumption. Some improvement may also be possible by reducing the 1-second dead time between magnet steps to a smaller value. Dead times of $200-300 \mathrm{~ms}$ have been tested and found to produce high-quality mass spectra.

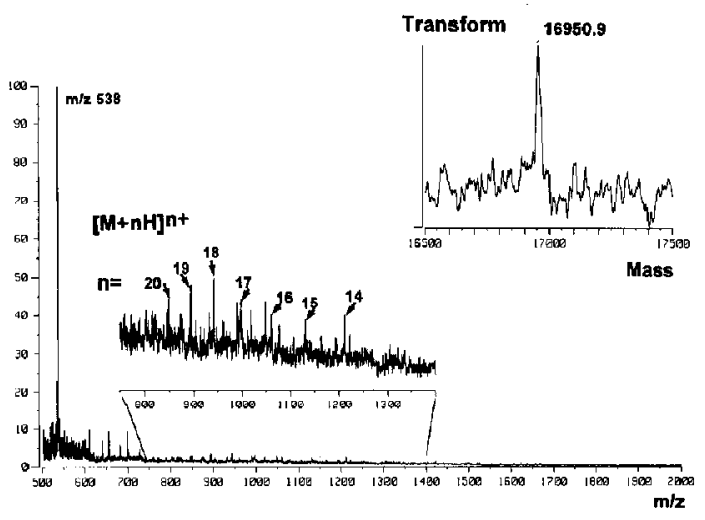

Figure 5. ESI mass spectrum acquired with the array detector for a $10 \mu \mathrm{l}$ loop injection of a $44 \mathrm{fmol} / \mu \mathrm{L}$ solution of myoglobin. Inset: Transformation of the mass spectrum by the "deconvolution" algorithm of Mann et al. [3]. 


\section{References}

1. Dole, M.; Mach, L. L.; Hines, R. L.; Mobley, R. C.; Ferguson, L. P.; Alice, M. B. J. Chem. Phys. 1968, 49, 2240.

2. Mach, L. L.; Kralik, P.; Rheude, A.; Dole, M. I. Chem. Phys. 1970, 52, 4977 .

3. Mann, M.; Meng, C.-K.; Fenn, J. B. Anal. Chem. 1989, 61, $1702-1708$.

4. Wong, S. F,; Meng, C.-K.; Fenn, J. B. J. Phys. Chem. 1988, 92, 546 .

5. Fenn, J. B.; Mann, M.; Meng, C.-K,; Wong, S. F.; Whitehouse, C. M. Science 1989, 246, 64 .

6. Fenn, J. B.; Mann, M; Meng, C.-K.; Wong, S. F.; Whitehouse, C. M. Mass Spectrom. Rev. 1990, 9, 37.

7. Meng., C.-K.; Mann, M.; Fenn, J. B. Z. Phys. D. 1988, $10,361$.

8. Covey, T. R,; Bonner, R. F.; Shushan, B. I.; Henion, J. Rapid Comm. Mass Spectrom. 1988, 2, 249-256.

9. Chowdhury, S. K.; Chait, B. T. Anal. Chem. 1991, 63, $1660-1664$.

10. Verentchikov, A. N.; Ens, W.; Standing, K. G. Presented at the 41st Annual ASMS Conference on Mass Spectrometry and Allied Topics; San Francisco, CA, May 31-June 4, 1993.

11. Berkel, G. J. V.; Glish, G. L.; McLuckey, S. A. Anal. Chem. $1990,62,1284-1295$.

12. Henry, K. D.; Williams, E. R.; Wang, B. H.; McLafferty, F. W.; Shabanowitz, J.; Hunt, D. F. Proc. Natl. Acad. Sci. USA 1989, 86, 9075-9078.

13. Loo, J. A,; Quinn, J. P.; Ryu, S. I.; Henry, K. D.; Senko, M. W.; McLafferty, F. W. Proc. Natl. Acad. Sci. USA 1992, 89, 286-289.

14. Henry, K. D.; Quirn, J. P.; McLafferty, F. W. J. Am. Chem. Soc. $1991,113,5447-5449$.

15. Limbach, P. A.; Grosshans, P. B.; Marshall, A. G. Anal. Chem. $1993,65,135-140$.

16. Guan, Z; Hofstadler, S. A.; Laude, D. A., Jr. Anal. Chem. 1993, 65, 1588-1593.

17. Meng, C.-K.; McEwen, C. N.; Larsen, B. S. Rapid Commun. Mass Spectrom. 1990, 4, 147-150.

18. Meng, C.-K.; McEwen, C. N.; Larsen, B. S. Rapid Commun. Mass Spectrom. 1990, 4, 151-155.

19. Gallegher, R. T.; Chapman, J. R.; Mann, M. Rapid Commun. Mass Spectrom. 1990, 4, 369-372.
20. Larsen, B. S.; McEwen, C. N. J. Am. Soc. Mass Spectrom. 1991, 2, 205-211.

21. Wada, Y.; Tamura, J.; Musselman, B. D.; Kassel, D. B.; Sakurai, T.; Matsuo, T. Rapid Cammun. Mass Spectrom. 1992, 6, 9-13.

22. Cody. R. B.; Tamura, J.: Musselman, B. D. Anal. Chem. 1992, 64, 1561-1570.

23. Cody, R. B. Proceedings of the 40th Amnual ASMS Conference on Mass Spectrometry and Allied Topics; Washington, DC, May 31-June 5, 1992; pp 1679-1680.

24. Dobberstein, P.; Schroder, E.; Munster, H. Prockedings of the 40th Annual ASMS Conference on Mass Spectrometry and Allied Topics; Washington, DC, May 3I-June 5, 1992; pp 1683-1684.

25. Bateman, R. H.; Bordoli, R. S.; Cashmore, P.; Major, H. J.; Woolfitt, A. R, Procedings of the 40th ASMS Conference on Mass Spectronetry and Nlied Topics; Washington, DC, May 31-Jtune 5, 1992; pp 1669-1670.

26. Wahl, J. H.; Goodlett, D. R.; Udseth, H. R.; Smith, R. D. Anal. Chem. 1992, 64, 3194-3198.

27. Mordehai, A.; Henion, J. D. Proceedings of the 40th Annual ASMS Conference on Mass Spectrometry and Allied Topics; Washington, DC, May 31-June 5, 1992; pp 197-198.

28. Ding. J.-M.; Anderegg, R. J.; Kassel, D. K. Proceedings of the 40th Annual ASMS Comference on Mass Spectrometry and Allied Topics; Washington, DC, May 31-June 5, 1992; pp 1671-1672.

29. Hill, J. A.; Biller, J. E.; Biemann, K. Int J. Mass Spectrom. Ion Proc. 1991, 111, 1 30.

30. Ishihara, M.; Kammei, Y. Rapid Commun. Mass Spectrom. 1989, 3,420 .

31. Hill, J. A.; Biller, J. E.; Martin, S. A.; Biemann, K. Int J. Mass Spectrom. Ion Proc., 1989, 92, 211.

32. Cotrell, J. S.; Evans, S. Anal. Chem. 1987, 59, 1990.

33. Tyler, A. N.; Romo, L. K.; Frey, M. H.; Musselman, B. D.; Tamura, J.; Cody, R. B. J. Am. Soc. Mass Spectrom. 1992, 3, 637-643.

34. Zaia, J.; Annan, R. S.; Biemann, K. Rapid Commun. Mass Spectrom. 1992, 6, 32-36.

35. Reinhold, B, B,; Rẹinhold, V. N. J. Am. Soc Mass Spectrom. 1992, 3, 20\%-215. 